\title{
Gestion des deux langues en classe bilingue : analyse comparative en Alsace et en Bretagne
}

Aurélie Mathes-Heintz et Anemone Geiger-Jaillet

\section{OpenEdition}

Édition électronique

URL : https://journals.openedition.org/esp/4365

DOI : $10.4000 /$ esp.4365

ISSN : 2532-0319

Éditeur

Centre d'Information sur l'Éducation Bilingue et Plurilingue

Édition imprimée

Date de publication : 1 décembre 2019

Pagination : 16-28

ISSN : 1127-266X

\section{Référence électronique}

Aurélie Mathes-Heintz et Anemone Geiger-Jaillet, « Gestion des deux langues en classe bilingue :

analyse comparative en Alsace et en Bretagne », Éducation et sociétés plurilingues [En ligne], 47 | 2019, mis en ligne le 01 septembre 2021, consulté le 01 septembre 2021. URL : http://

journals.openedition.org/esp/4365; DOI : https://doi.org/10.4000/esp.4365 


\section{GESTION DES DEUX LANGUES EN CLASSE BILINGUE: ANALYSE COMPARATIVE EN ALSAGE ET EN BRETAGNE}

\section{Aurélie Mathes-Heintz, Anemone Geiger-JAillet}

L'insegnamento bilingue francese-lingua regionale nel ciclo primario richiede allinsegnante di mettere in atto pratiche pedagogiche particolari al fine di insegnare in lingua regionale ad alunni spesso principianti nella lingua regionale in questione. La ricerca di pratiche didattiche dei professori che insegnano nelle due lingue nelle loro classi (1 insegnante - 2 lingue) in Alsazia e in Bretagna mette in luce pratiche, ma anche motivazioni mollo diverse nelle due regioni. Questo studio permelle di rivedere il posto della L1 e più in generale la visione stessa di quello che è un insegnamento bilingue, piuttosto considerato come due monolinguismi paralleli in Alsazia e trattato invece in un'ottica più bi-plurilingue in Bretagna.

Parole chiave: insegnamento bilingue francese - lingua regionale (Bretagna, Alsazia), immersione precoce, 1 insegnante - 2 lingue (1 maestro - 2 lingue), code-switching, posto della L1.

Zusammenfassung: In Frankreich gibt es in verschiedenen Regionen seit 40 Fahren die Möglichkeit, zweisprachig mit Französisch neben der lokal vorhandenen Regionalsprache beschult zu werden. Während mehrheitlich ein Lehrer für eine Sprache zuständig ist, gibt es auch Fälle von Lehrkräften, die in beiden Sprachen in ihrer Klasse unterrichten. Die vorliegende Studie hinterfragt, wie in der Bretagne und im Elsass die Erstsprache während des Unterrichts in der Regionalsprache eingesetzt und wie zweisprachiger Unterricht angesehen wird. Dieser scheint im Elsass eher wie parall geführter monolingualer Unterricht in zwei Sprachen zu sein, während in der Bretagne die Zweisprachigkeit im Unterricht eher als Fundament von Zwei- oder Mehrsprachigkeit dient.

Schlüsselwörter: Zweisprachigkeil im Unlerricht Französisch - Regionalsprache (Brelagne, Elsass), früher Fremdsprachenerwerb, ein Lehrer - zwei Sprachen, Sprachwechsel (code-switching), Platz von L1.

PARTICULARITÉS DES CLASSES

BILINGUES SEMI-

IMMERSIVES
Q uel bilinguisme dans les classes bilingues? Le professeur des écoles en classe bilingue enseigne les disciplines en langue régionale à des élèves pour qui cette dernière s'apparente bie-souvent à une langue étrangère. Il s'agit donc, pour les élèves, d'un bilinguisme en construction ou en devenir. Il est aisé de comprendre que le professeur ayant en charge la partie des enseignements en langue régionale se retrouvera confronté à un certain nombre de problématiques d'ordre pédagogique et didactique dues à cette réalité. Dans les sites bilingues, c'est donc bien l'éducation qui est bilingue, dans le sens où une seconde langue est utilisée comme moyen de transmission de contenus disciplinaires. Les élèves inscrits en classe bilingue sont, eux, rarement bilingues au départ de leur 


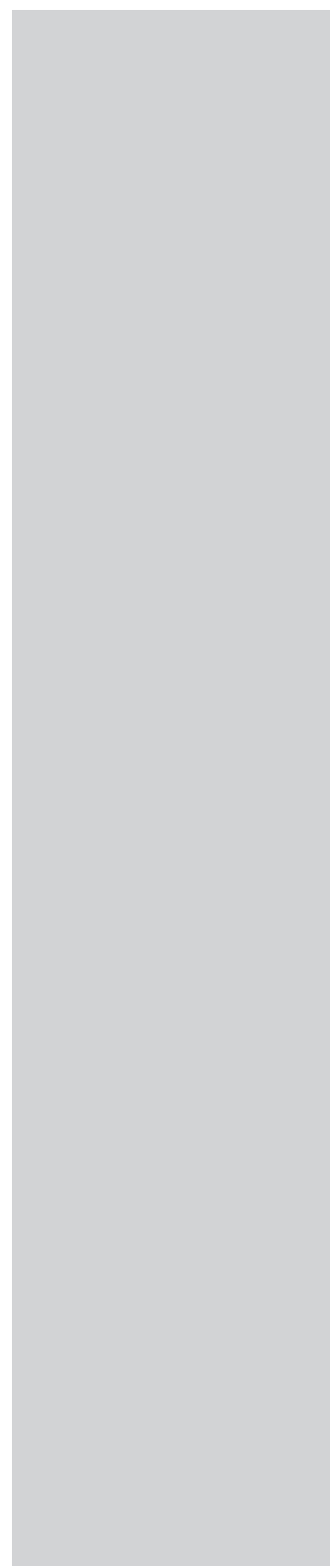

scolarité en maternelle.

Cependant, il peut paraitre surprenant que dans ces classes dites «bilingues», c'est souvent une seule langue qui est utilisée pendant une séance, une demi-journée ou même une journée d'enseignement. En effet, le système d'enseignement bilingue paritaire par immersion, tel qu'il est actuellement pratiqué en Alsace, par exemple, est comparable à deux enseignements distincts réalisés par deux professeurs. Dans la grande majorité des cas, l'enseignement en langue allemande est assuré, selon l'une des deux modalités proposées dans les textes officiels (MEN 2001: BO N³3), par un professeur alors que l'enseignement en langue française sur l'autre moitié du temps scolaire est assuré par un autre enseignant sclon le principe «un enseignant - une langue». Dans ce cas, l'enseignement se caractérise par un monolinguisme en langue française juxtaposé à une autre en langue régionale. Il en résulte donc bien souvent, dans la pratique, deux formes d'enseignement cloisonnées, sans lien entre elles.

Il s'agit bien là d'un phénomène que pointent bon nombre de recherches sur ces systèmes immersifs en alternance, pas uniquement en Alsace. Beardsmore (2010: 103) soulève par exemple que «la formation des enseignants en éducation bilingue représente une lacune dans la plupart des pays et constitue donc un défi important à relever» et les chercheurs Gajo et Berthoud (2008: 9) dénoncent «deux monolinguismes en parallèle» car le bilinguisme tel qu'il est pratiqué actuellement dans bon nombre de systèmes a tendance à scinder les langues plus qu'à les rapprocher. Ils sont rejoints par Castellotti et Moore sur ce point:

L'enseignement des langues reste envisagé le plus souvent sinon sur le mode de la stricte séparation, du moins sur celui de l'ignorance mutuelle, ce qui est renforcé par l'organisation institutionnelle de la plupart des systèmes éducatifs européens qui fonctionnent sur le principe du cloisonnement disciplinaire (Castellotti, Moore 2002: 16).

Or en Alsace, malgré la prédominance du modèle «une personne une langue», un certain nombre d'enseignants bilingues assure l'enseignement dans les deux langues au sein du même groupe-classe. Sclon un article des Dermières Nowvelles d'Alsace du 28 mai 2016 (Clerc 2016), il y avait quarante-sept classes en 2015-16, soit 8,8\% des enseignements de classes bilingues travaillant selon ce principe. Même s'il ne s'agit pas de la pratique la plus courante dans les classes bilingues en Alsace, ce phénomène peut offrir un terrain d'investigation.

Il existe d'autres régions françaises avec un tel enseignement bilingue régional. A l'exemple de la Bretagne ou de l'Occitanie, celui-ci peut également être assuré par la même personne dans les deux langues ce 


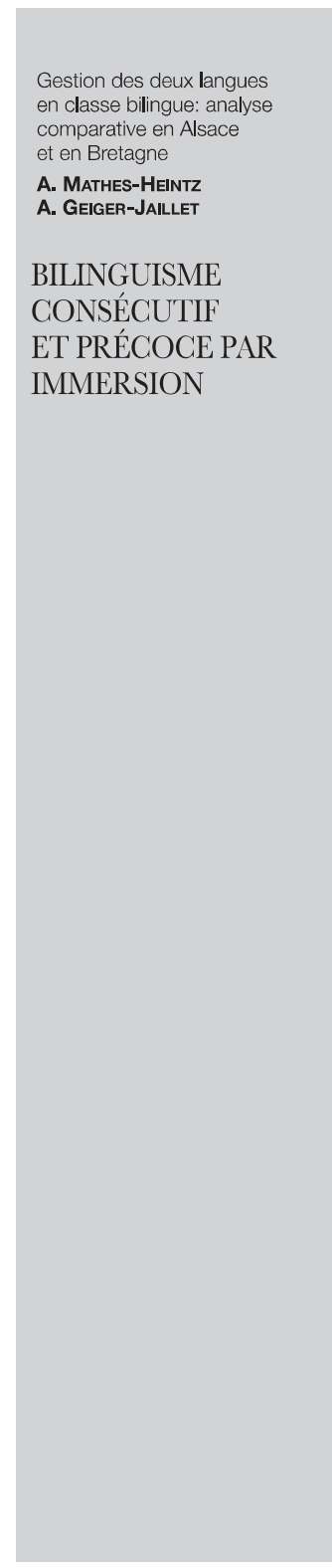

qui amène à des changements de langue. Appelé également codeswitching, ces pratiques de l'alternance codique dans l'enseignement primaire sont une réalité au Luxembourg et dans la Vallée d'Aoste par exemple. Notre démarche sera alors comparative.

Quels avantages et quelles spécificités dans le cadre scolaire? Il est aujourd'hui démontré que le bilinguisme ou la pratique d'une langue étrangère en bas âge ne se fait pas au détriment de la langue maternelle ou de la langue première, même si celle-ci est encore en construction (Hélot, Rubio 2013: 46-47), à condition qu'un certain nombre de facteurs soient réunis comme le prestige égal des langues en question et surtout l'attitude de la famille, de l'entourage et de l'enfant lui-même par rapport à ce bilinguisme et aux langues en question (Geiger-Jaillet 2005: 27-28). Les recherches actuelles démontrent que les différentes langues en présence peuvent se coconstruire, notamment par le biais d'activités cognitives métalinguistiques que le locuteur met en œuvre (Kail 2015: 92), et même, que «[...] l'acquisition précoce et intensive d'une langue 2 aboutit à une maitrise plus poussée de la langue maternelle» (Petit 2001: 48). Ces avantages sont rappelés dans les textes officiels concernant l'enseignement bilingue: «tout en permettant la transmission des langues régionales, il conforte l'apprentissage du français et prépare les élèves à l'apprentissage d'autres langues» (MEN 2001: BO N³3).

D'autres études démontrent également la plus grande facilité des bilingues à apprendre d'autres langues et d'une façon plus générale à faire preuve d'abstraction. On ne niera donc plus les bienfaits du biou du plurilinguisme, même en bas âge, qui sont maintenant amplement attestés. L'enfant, en âge préscolaire et en classe immersive construit son langage et apprend en même temps une langue régionale, souvent étrangère pour lui. Mais il s'agit bien souvent, ni de la langue de la famille, ni de celle qu'il peut entendre dans un autre contexte social. Le jeune enfant est donc exposé à une situation «artificielle» où seul l'enseignant parle cette langue. La motivation pour son apprentissage n'est pas guidée par la nécessité de communiquer pour survivre, comme le serait l'apprentissage de la langue maternelle. Il ne s'agit pas non plus d'un bilinguisme familial qui se construit en interaction et avec toutes les dimensions affectives et culturelles qu'une langue peut apporter. Cette méthode d'apprentissage par immersion est également loin d'être «naturelle», au sens où l'enfant apprendrait sans effort et sans difficultés. Au contraire, cette pratique pédagogique demande un certain nombre de facultés cognitives et attentionnelles de la part de l'élève. Geiger-Jaillet (2005; 2016) met également en garde contre cette idée qui sous-entendrait que le bilin- 


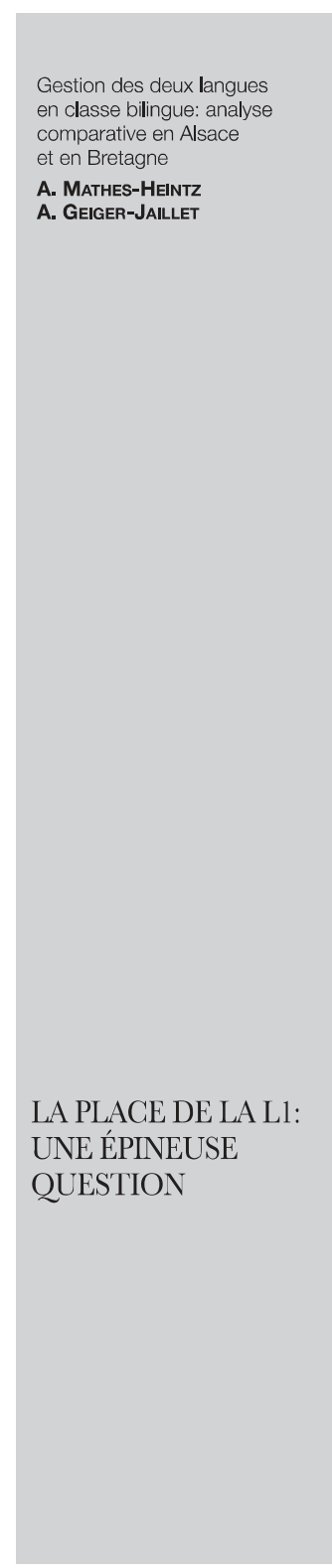

guisme acquis par immersion, qualifié parfois de méthode «naturelle», s'acquerrait «sans effort» (Geiger-Jaillet 2005; 2016).

Pour permettre à un enfant bilingue de naissance d'atteindre un bilinguisme équilibré, beaucoup d'efforts et de stratégies parentales doivent être mises en place afin qu'il puisse maîtriser le plus de situations de communications possibles dans les deux langues. Il en est de même pour le bilinguisme scolaire: mettre son enfant dans une école bilingue et s'attendre à ce que la fréquentation seule permette à l'enfant de devenir bilingue risque de mener à des mécomptes ou à des désillusions. (Geiger-Jaillet 2005: 23)

Il s'agit bien d'une situation tout à fait particulière pour laquelle la motivation de l'apprenant et de son entourage sera l'une des conditions sine qua non de la réussite.

Un autre point important à souligner est que le bilinguisme par immersion en milieu scolaire a ses particularités propres, puisque les interactions communicationnelles sont, en grande partie, limitées au domaine scolaire. Le cadre même de l'école, comme seul vecteur d'apprentissage de la langue, est également particulier, puisque les situations communicationnelles sur le plan scolaire sont spécifiques. Colin (2012: 449) note que «si les interactions sont certes authentiques, avec des buts communicatifs réels, elles restent néanmoins très différentes des conditions d'interaction en famille, notamment en raison de la structure de l'interaction scolaire (dimension collective des échanges)». De plus, elles n'offrent pas suffisamment de situations d'interactions orales pour que la langue puisse se développer de la même façon qu'avec des pairs natifs

Ainsi, l'apprentissage d'une langue étrangère par immersion en contexte scolaire pour de jeunes élèves se révèle d'une certaine façon «artificielle», et fortement dépendant de la motivation et de la persévérance du jeune élève et de son entourage.

Comme le précise Duverger (2009), dans le cadre d'une conférence en ligne tenue à l'occasion du séminaire bilingue sur le thème du statut de la langue cible et de la langue maternelle dans l'enseignement des disciplines dites non linguistiques: «Quand on enseigne en L2, la L1 n'est jamais absente, même si seule une des deux langues apparaît». Mềme si le recours à la L1 (souvent le français) par l'ćlève des classes bilingues en immersion est généralement toléré, ce passage à la langue commune de référence par le professeur n'est, par contre, pas préconisé, même en petite section.

Finalement, la pratique du bilinguisme soulève l'épineuse question de la place de la langue première dans les apprentissages. Pourquoi, alors que pour un enfant allophone le recours à la L1 serait encouragé afin qu'il établisse des liens avec sa langue, n’en serait-il pas de 


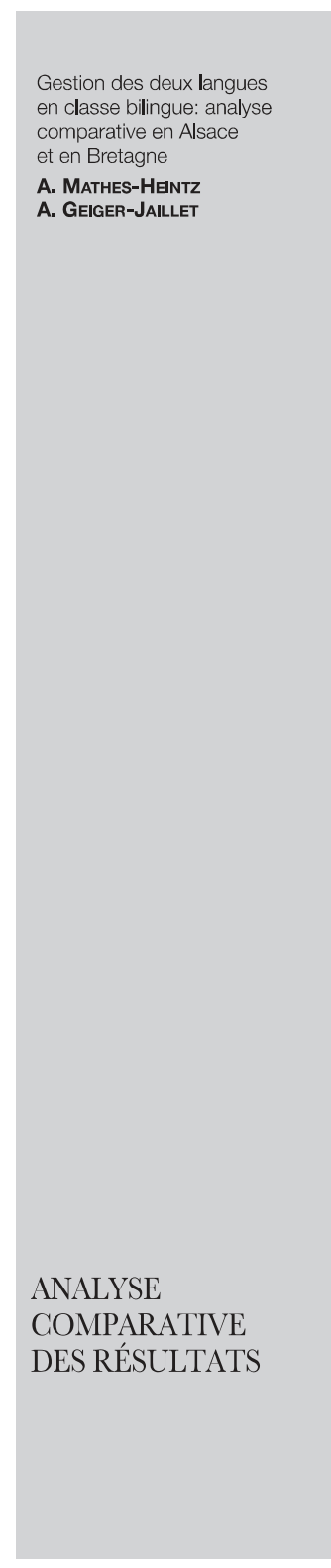

même pour un apprenant en immersion? Ceci est probablement due à la tradition monolingue de nos sociétés européennes et à une certaine vision «puriste» des langues où l'alternance, la micro-alternance ou le mélange des langues n'ont pas très bonne réputation et la question semble même un peu taboue. "Longtemps négligé par la recherche et dénigré par la société comme preuve d'incompétence, le code-switching a récupéré un droit de cité dans les études linguistiques et sociolinguistiques [...]» (Lüdi 2004: \$21). Concernant le code-switching, Kail (2015: 14) précise que «ce phénomène longtemps stigmatisé a plus récemment fait l'objet de nombreuses recherches [...] [et] il est maintenant admis que ce processus remplit des fonctions précises dans les stratégies de communication». Cependant, du point de vue individuel, on peut ressentir un certain malaise face à cette question lorsqu' elle se pose, comme si elle dérangeait. Gajo et Berthoud (2008) relèvent également «un certain malaise» lorsque la question de la micro-alternance codique se pose.

Que dire alors de l'enseignement par immersion? Une alternance des langues est-elle envisageable? Comment mettre en relation les deux langues? La question est délicate et rarement abordée:

Dans la problématique de l'enseignement bilingue, un des aspects les moins étudiés et les plus controversés est certainement celui de la relation entre Ll et L2. On se retrouve très vite face à une visée immersive «dure» qui préconise l'acquisition de L2 par le fait même d'exclure L1 et qui craint l'alternance des langues et le mélange hybride qui en résulterait (Gajo, Berthoud 2008: 9).

Force est de constater que les positions sont contrastées à ce niveau, surtout en ce qui concerne le recours à la Ll par l'enseignant. Quelles sont les pratiques pédagogiques dans nos classes bilingues? Comment se caractérisent les pratiques enseignantes des professeurs qui enseignent dans les deux langues (français et langue régionale) selon le principe 1 enseignant-2 langues? S'agit-il de deux monolinguismes juxtaposés ou d'une véritable pratique et didactique bilingue? Les apports de notre recherche nous permettent-elles de dresser un état des lieux des pratiques enseignantes dans les classes bilingues en Alsace et en Bretagne?

Dans le cadre d'un mémoire de master (Mathes-Heintz 2017), un questionnaire en ligne a été envoyé aux directeurs des écoles offrant une section bilingue, en précisant que celui-ci s'adressait aux enseignants qui enseignent en français et en langue régionale dans leur classe, selon le principe «l maitre - 2 langues»: 68 enseignants ont répondu pour l'Alsace, 27 pour la Bretagne. Pour information, cette forme d'organisation «lMlL» est la moins répandue en Alsace, alors qu'elle est majoritaire en Bretagne, mais le phénomène y concerne 


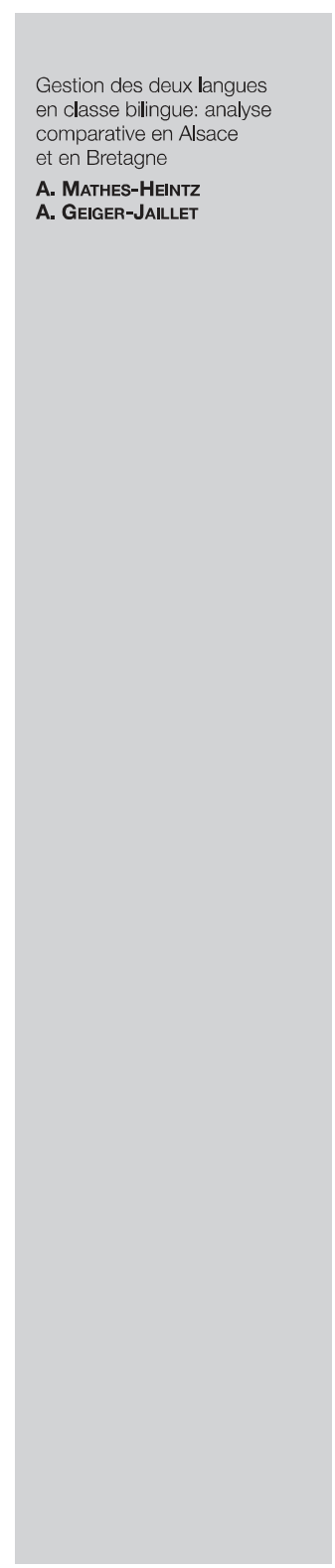

moitié moins d'élèves en chiffres absolus. Environ 20\% des élèves de maternelle fréquentent des sites bilingues paritaires à la rentrée 2017 18 dans l'académie de Strasbourg et $17 \%$ de la population scolaire à l'école primaire, ce qui fait près de 29.000 élèves (https://www.acstrasbourg.fr/delecoleausuperieur/cursus-bilingue-allemand/). Dans l'académie de Rennes, environ $11,7 \%$ de la population scolaire est concernée par cet enseignement bilingue français-breton ce qui fait 14.656 élèves (privé et public) à la rentrée 2018 (Dossier de presse rentrée scolaire 2018-19, effectif total premier degré: 124.750 élèves; http://cache.media.education.gouv.fr/file/presse/37/1/DP_rentree Acad_2018-2019_990371.pdf).

Le questionnaire était organisé en deux partics. La première partic intitulée "Vous et votre environnement éducatif» permettait de recueillir des informations générales sur les enseignants et leur environnement scolaire. La deuxième partie, à partir de la question 15, intitulée «Vous et l'enseignement bilingue en pratique», renseignait sur les pratiques pédagogiques en classe.

Dans les deux échantillons, les écoles présentaient à peu près les mêmes caractéristiques (plus de 7 classes, avec souvent plusieurs enseignants bilingues au sein de l'école, enseignant majoritairement en double-niveau). Ils représentent des enseignants des trois cycles de l'école primaire. En Alsace, les enseignants des deux premiers cycles ont davantage répondu à cette étude (cycle 1: 27 sur 67; cycle 2: 26 sur 67; cycle 3: 17 sur 67 et certains enseignants à cheval sur deux niveaux); en Bretagne, ce sont les enseignants des cycles 2 et 3 qui sont les plus représentés (cycle 1: 7 sur 24; cycle 2: 13 sur 24; cycle 3: 9 sur 24 répondants).

Il s'agissait de brosser un tableau des pratiques pédagogiques concernant la gestion des deux langues dans leurs classes, et de recueillir des indicateurs sur leurs motivations et leurs convictions personnelles concernant le bilinguisme de façon plus générale. Le questionnaire s'adressait aux enseignants bilingues qui enseignent en français ET en langue régionale dans deux académies de la Métropole - qu'ils soient novices ou non.

Dans l'Académie de Strasbourg, nous avons questionné les enseignants de l'école primaire enseignant selon le principe «un maitredeux langues» qui est la forme d'organisation la moins répandue. Dans un souci de comparaison des pratiques des enseignants bilingues, nous avons inclus des enseignants en sites bilingues de l'académie de Bretagne, qui enseignent selon ce même principe, avec deux différences: la Bretagne a une expérience du bilinguisme scolaire plus longue que l'Alsace et le principe d'un maitre transmettant les savoirs et connaissances en deux langues y est plus répandue. 


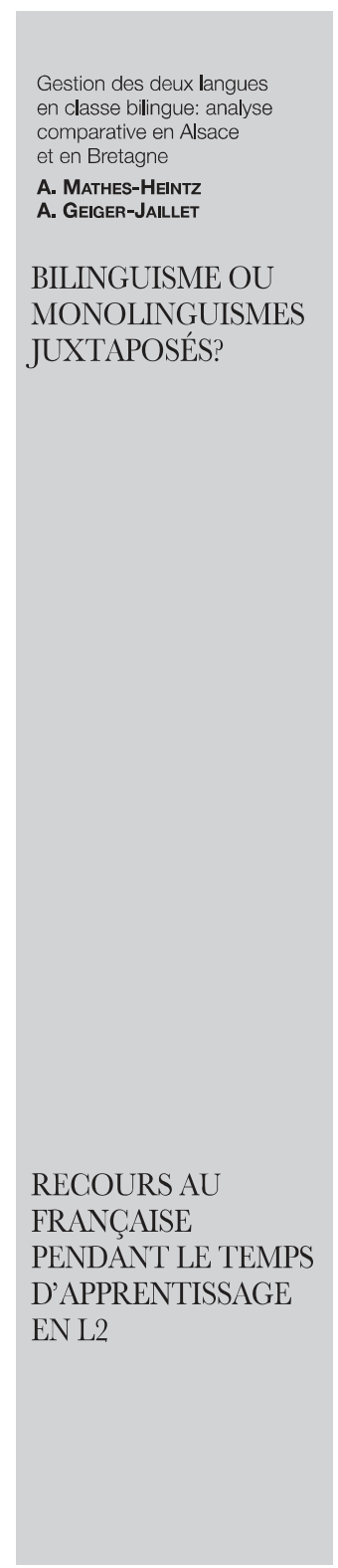

La gestion des deux langues dans les classes bilingues s'organise de façon bien différente en Alsace et en Bretagne. En Alsace, la préoccupation principale semble tourner autour de la gestion du temps. Toutes les formes d'organisation reflètent une stricte application de l'horaire pour chacune des langues, un «cloisonnement» des langues que dénoncent d'ailleurs certains chercheurs (Gajo, Berthoud 2008; Böing 2011). La première hypothèse que nous pouvons avancer (Mathes-Heintz 2017) est que les professeurs des écoles bilingues en Alsace ont peut-être été davantage sensibilisés à la notion de «parité horaire» à respecter dans les deux langues, d'autant plus que la majorité d'entre eux a enseigné uniquement en langue allemande avant d'enseigner dans les deux langues, ce qui n'est absolument pas le cas en Bretagne d'après les réponses apportées. Il est possible que les enseignants appliquent donc cette stricte gestion du temps pour les deux langues par commodité, par habitude, ou pour permettre la stricte application des textes officiels. Il faut rappeler que dans l'enseignement public en France, l'apport de la langue régionale ne peut pas dépasser 50\% du temps d'enseignement d'une semaine. Il est donc très fréquent qu'un enseignant fasse deux mi-temps d'enseignement dans deux classes différentes.

Si en Alsace la gestion des langues s'apparente plutôt à un cloisonnement monolingue dans un cadre horaire strict, en Bretagne, la pratique de classe semble moins cloisonnée et l'alternance concernerait des matières ou des séances d'apprentissage. Cependant, d'autres formes d'organisation émergent également en Bretagne, même si elles sont minoritaires. Elles correspondent à une vision immersive quasi-complète en langue régionale afin de permettre un maximum d'acquisition dans la langue la moins présente dans l'environnement extra-scolaire. Le français reste alors limité aux activités culturelles et langagières en langue française. Ces enseignants semblent plutôt engagés dans une démarche de sauvegarde du patrimoine culturel et linguistique, ce qui explique probablement cette gestion des langues.

Maintenant que nous pouvons nous faire une idée de l'articulation des deux langues dans les classes au niveau macro, voyons si, quand, et à quelle fréquence, la langue française est employée en-dehors des espaces temps lui ćtant réscrvés. Car, «dans la problématique de l'enseignement bilingue, un des aspects les moins étudiés et les plus controversés est certainement celui de la relation entre L1 et L2» (Gajo, Berthoud 2008: 9). Les deux langues en présence dans un système dit bilingue et dont l'enseignement est assuré par le même enseignant, devra permettre d'étudier cette question.

La question du recours à la langue française au sein d'une séance d'apprentissage menée en langue régionale a été posée de manière 


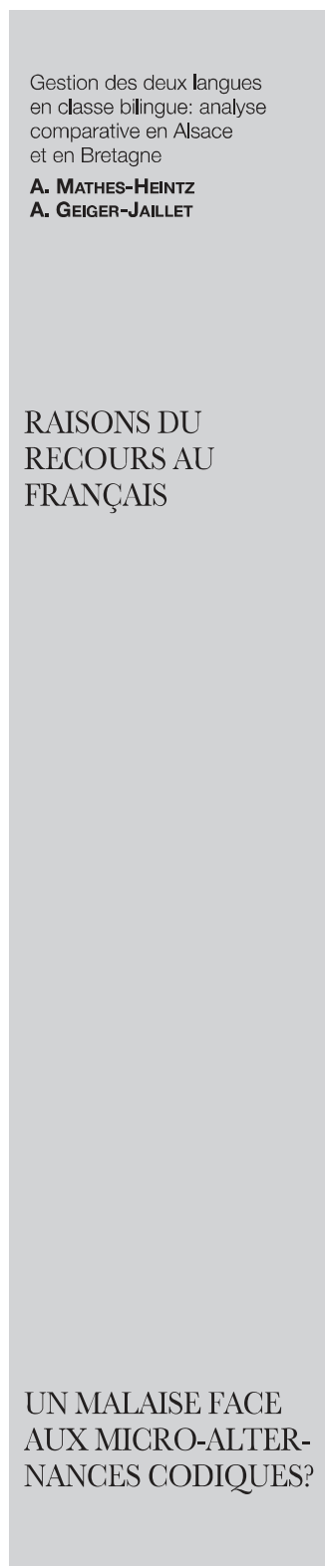

identique aux enseignants du corpus breton et à ceux en Alsace et a fait ressortir d'autres différences. La majorité des enseignants en Alsace ayant répondu à cette question (17 non-réponses cependant) dit y recourir parfois (26 sur 51), et 14 disent y recourir souvent. En Bretagne par contre, la moitié des sondés dit y recourir rarement 13 sur 26) et 11 enseignants parfois.

Le recours à la langue française interviendrait donc plus fréquemment en Alsace qu'en Bretagne, mais en réponse à la question de savoir pourquoi, 17 sur 51 enseignants en Alsace n'ont pas répondu et la question reste en suspens et traduit peut-être un malaise.

Notons à présent les raisons avancées par les enscignants alsaciens pour justifier le recours à la langue française pendant une séance initialement prévue en langue régionale. En Alsace, sur les 68 enseignants bilingues ayant participé à l'enquête, 17 ne se sont pas prononcés sur cette question.

Le questionnaire proposait plusieurs choix qui motivent le recours au français par l'enseignant: la motivation indiquée le plus souvent est liée aux problèmes de comportements, de règles de vie de la classe, donc au fonctionnement de la classe elle-même. Cette raison a été mentionnée 40 fois. La deuxième raison d'un changement de langue est de faire comprendre un concept mal compris à un élève (raison mentionnée 35 fois). Il s'agirait donc d'une «alternance d'usage ordinaire»(Steffen 2013: 108) dans les deux premiers cas. La troisième raison avancée, mentionnée 20 fois, est celle en vue de comparer les langues et leur fonctionnement ce qui classerait ce changement comme une «alternance d'apprentissage».

Voyons à présent le recours à la langue française pendant une séance initialement prévue en langue régionale par les enseignants de Bretagne. Les 26 réponses fournies permettent d'avancer que la majorité des réponses bretonnes suggère une réflexion métalinguistique et presque une stratégie de leur part. Dans le détail, la réponse qui revient le plus souvent est celle de comparer les deux langues et leur fonctionnement respectif ou leur symbolique (mentionnée 17 fois), combinée à celle de se sortir d'une impasse linguistique (raison mentionnćc 10 fois), suivic de la troisième raison avancéc (mentionnéc 9 fois) qui est celle du fonctionnement de la langue.

Le nombre important de non-réponses du côté des enseignants alsaciens pose question, parce que presque un tiers des participants alsaciens n'a pas répondu à la question de la gestion des langues dans sa classe. Il est peu probable que la question ait été mal posée, puisqu'en Bretagne elle n'a obtenu qu'une seule non-réponse. Est-ce que le 


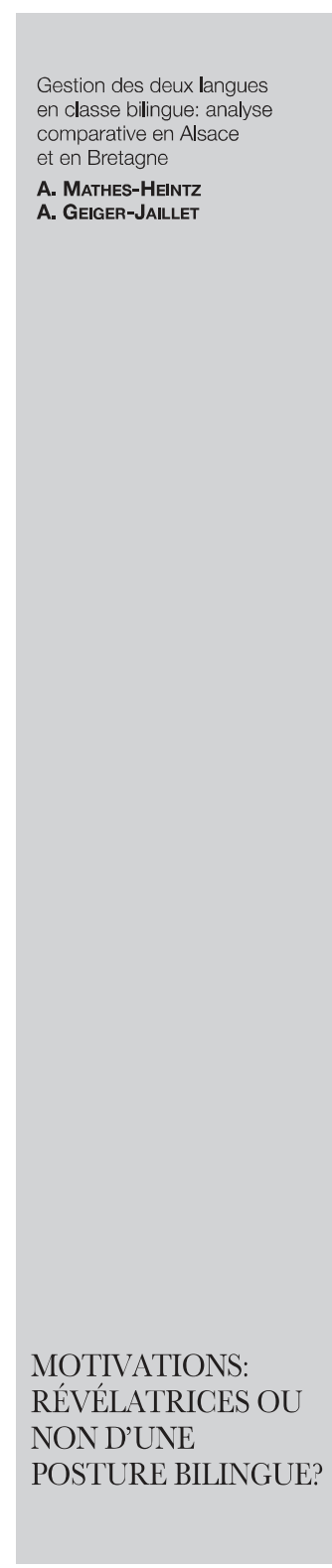

nombre de non-réponses serait dû à une erreur d'aiguillage du questionnaire, cette partie ne concernant que les professeurs qui enseignent dans les deux langues? Il est possible que certains enseignants, se rendant compte qu'ils ne sont pas concernés par les questions posées à partir de Q16, n'aient plus répondu. Force est de constater qu'à partir de cette question, 15 enseignants n'ont plus répondu jusqu'au bout de l'enquête. Ou alors est-ce effectivement la (micro-)gestion des langues qui pose problème car, comme le soutiennent certains chercheurs, ces enseignants se sentiraient peu à l'aise face à ce sujet? Les non-réponses traduiraient alors ce malaise. Ne sachant pas comment répondre à la question, par pudeur ou par crainte de ne pas être en adéquation avec ce qu'on pourrait leur demander, certains enseignants ont peut-être préféré ne pas répondre. La communication sur ce sujet est en effet plus transparente en Bretagne, où une note de cadrage sur les enseignements en classe bilingue existe. L'absence d'une telle modalité peut contribuer à accentuer le climat d'incertitude sur le sujet qui semble transparaittre au travers des réponses alsaciennes. Il semblerait donc que ces questions de gestion des langues posent plus problème au niveau du bilinguisme en Alsace, alors qu'elles semblent moins taboues en Bretagne. Rappelons tout de même que cela peut également être lié aux modalités de mise en œuvre du bilinguisme. Les postes «1 maitre-2 langues» sont minoritaires en Alsace, alors qu'il s'agit plutôt de la norme en Bretagne. Au vu des difficultés de recrutement en Alsace, cela peut facilement s'expliquer. En effet, en affectant les professeurs qui enseignent en langue allemande à une classe au lieu de deux, cela revient concrètement à perdre un demi-poste en langue régionale pour l'administration scolaire. Au vu de la pénurie des professeurs sachant enseigner en langue régionale, ce critère a évidemment son importance. D'après les informations que nous avons pu obtenir, la Bretagne, ayant fait des efforts de revitalisation de la langue y compris au niveau universitaire depuis bien plus longtemps, ne serait plus confrontée aux mêmes problématiques dans le premier degré. Mais en termes de chiffres absolus d'élèves, le bilinguisme concerne certes moins d'élèves, moins de classes et par conséquent, moins de postes à pourvoir.

La motivation, nous l'avons vu plus haut, a son importance pour la réussite de ce dispositif pour l'élève et son entourage, mais aussi pour le professeur lui-même, pour qui la tâche n'est pas aisée. Lorsque l'on interroge les enseignants sur leur satisfaction à enseigner dans ce type de configuration (1 enseignant-2 langues), la grande majorité des enseignants y trouve satisfaction $(40 / 44$ en 


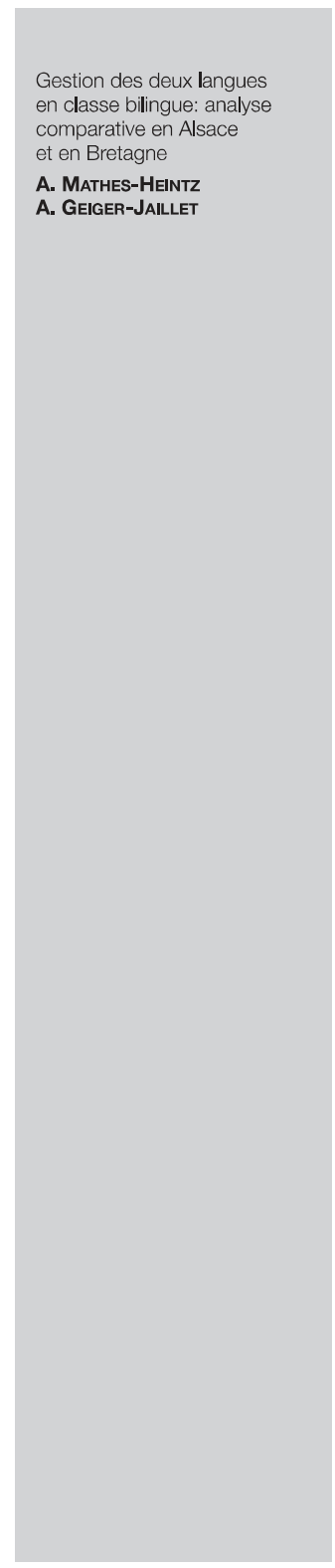

Alsace et 22/23 en Bretagne).

Cependant, les motivations qui ressortent pour ce type de poste sont différentes selon la région. Lorsque l'on interroge les enseignants sur leurs motivations à enseigner dans les deux langues en Alsace, elles semblent avant tout être d'ordre pratique avant d'être linguistiques. En effet, la réponse au choix de l'enseignement dans les deux langues («avoir sa propre classe ») revient le plus souvent. Gette réponse peut toutefois cacher des réalités diverses (organisation, langues, flexibilité...) et ne donne finalement pas tant d'informations. C'est la raison pour laquelle il est important de mettre en relation les réponses apportées aux deux questions posées: «Pourquoi enseignez-vous dans les deux langues?» et «Appréciezvous d'enseigner dans les deux langues?» Les autres raisons de la satisfaction des enseignants concernent le fait de pouvoir faire des liens entre les apprentissages et les disciplines, au cœur même des apprentissages scolaires, dans une sorte de posture bi- plurilingue de l'enseignant.

Dans le cas des enseignants bretons, le militantisme culturel et linguistique apparait clairement comme étant la première raison de ce choix d'enseignement. Toutefois, la question posée ne fait pas appel aux mêmes réalités culturelles et scolaires dans les deux régions. En effet, en Alsace, la question de la gestion des deux langues dans une classe s'entend par opposition au système plus généralisé du modèle «l maitre-1 langue», alors qu'en Bretagne ce modèle constitue plutôt l'exception. Il fallait donc en fait comprendre «Pourquoi enseignez-vous dans les deux langues plutôt que de n'être chargé que de la partie des enseignements en langue régionale? Quels avantages / inconvénients y voyez-vous?». En tenant compte de cela, les réponses côté breton ne peuvent évidemment pas être de même nature. Dans les motifs de satisfaction cités pour ce type d'enseignement, l'enrichissement, le plaisir d'être témoin de l'apprentissage du breton et la transmission de la langue sont les principaux. La dimension multilingue ou bilingue n'apparaît pas clairement ici. Dans les motifs d'insatisfaction, hormis la charge de travail supplémentaire, apparaissent aussi deux fois le regret que le breton ne puisse pas être la seule langue d'enseignement. Cette idée n’a été avancée par aucun enseignant en Alsace. Les motivations des enseignants bretons semblent donc être avant tout liées à la transmission de la langue, d'une culture, d'un patrimoine dont ils sont les garants. Il n'est donc pas étonnant que les enseignants bretons semblent moins enclins à utiliser la langue française dans leur enseignement. Nous pouvons par contre constater, au vu des réponses obtenues, que, lorsqu'ils ont recours au français, ils l'utilisent dans une 


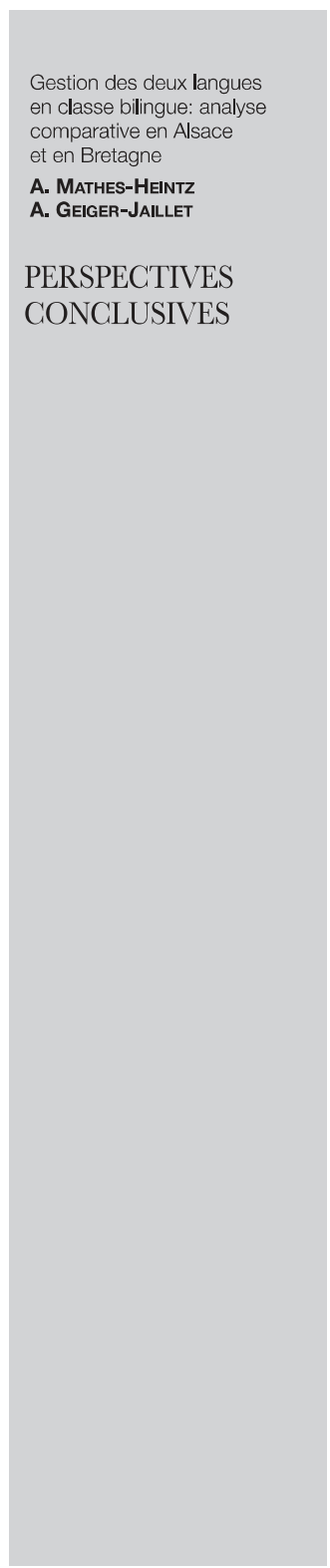

volonté de comparaison des langues dans la majorité des cas (cité dans 8 cas sur 13) et qu'ils sont donc là véritablement dans une posture bilingue.

Le but de cette recherche était de dresser un premier état des lieux des pratiques des professeurs des écoles enseignant dans les deux langues (français et langue régionale) dans leur classe afin de déterminer si leur enseignement se caractérise plutôt par un monolinguisme dans deux langues ou par une véritable pédagogie et pratique bilingues.

A la lumière des résultats obtenus pour les deux régions, nous pouvons dire que les pratiques et les motivations enscignantes y sont très différentes. Au niveau macro-organisationnel, la pratique de classe peut être qualifiée de bilingue en Bretagne car en effet, les deux langues sont utilisées pour les différentes phases d'apprentissage, les matières étant enseignées dans une langue ou dans l'autre, parfois dans les deux langues au cours d'une même séance. En revanche, en Alsace, la pratique semble plutôt indiquer deux monolinguismes juxtaposés. En effet, l'alternance des langues y est guidée par un cadre horaire strict dans lequel seule une seule langue aura sa place. Une étude ultérieure dans d'autres écoles bilingues françaises permettrait d'en dresser un tableau plus global.

A partir des incises en langue française pendant les apprentissages réalisés en L2, nous pouvons également déterminer la place des langues en classe et la posture bilingue ou non de l'enseignant. Ainsi, en Alsace, si le recours au français semble être plus fréquent qu'en Bretagne, il l'est surtout pour des raisons de gestion de la classe. A l'inverse, en Bretagne, le recours au français, moins fréquent, est avant tout utilisé pour des raisons qui permettent de mieux comprendre et de comparer les langues. Il s'agit là d'un positionnement, d'une posture de la part des enseignants que l'on peut également qualifier de bilingue. De plus, le recours à la L1 semble problématisé et assumé en Bretagne alors qu'en Alsace la question même de l'alternance codique a suscité un nombre important de non-réponses, traduisant ainsi un malaise.

Les raisons de ce bilinguisme scolaire apparemment plus abouti en Bretagne qu'en Alsace n'ont pas pu être expliquées par des différences dues à l'environnement scolaire, ni aux stéréotypes concernant l'apprentissage des langues ou du bilinguisme. Par contre, la valeur affective de la langue est un facteur important qui intervient en Bretagne, et qui est absent des réponses obtenues en Alsace. Cela est peut-être également dû à la situation de nombreux enseignants germanophones mais non alsaciens parmi les enseignants des classes 


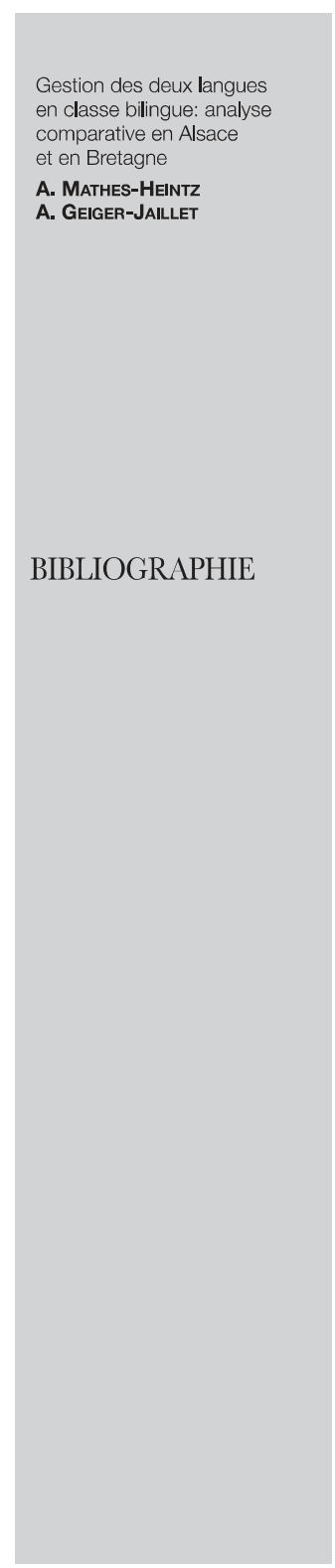

bilingues Cette valeur affective et davantage identitaire est certainement l'un des moteurs de cette motivation pédagogique bilingue en Bretagne. Néanmoins, il ne s'agit peut-être pas là de la seule explication. D'autres recherches devront se pencher sur la question de la formation professionnelle et de l'auto-formation après l'entrée dans le métier. Cela permettrait de savoir, par exemple si les formateurs intervenant dans ces dispositifs, questionnent la place des langues respectives dans un dispositif véritablement bilingue et le positionnement à avoir par rapport au contact de la langue régionale et du français.

BAETENS BEARDSMORE H. 2010. Le problème de l'unilinguisme dans la formation bilingue, 103-114 in Ehrhart S., Hélot C., Le Nevez A. (éds.) Plurilinguisme et formation des enseignants. Une approche critique. Plurilingualism and Teacher Education. A critical Approach. Frankfurt am Main: Peter Lang.

BÖING M. 2011. Comment aborder l'enseignement bilingue? Bilinguale Unterrichtsformen planen und durchführen. Der fremdsprachliche Unterricht Französisch, Heft 110, 2-8.

CASTELLOTTI V., MOORE D. 2002. Représentations sociales des langues el enseignements. Guide pour l'élaboration des politiques linguistiques éducatives en Europe - De la diversité linguistique à l'éducation plurilingue. Division des politiques linguistiques. Direction de l'éducation scolaire, extrascolaire et de l'enseignement supérieur. DGIV. Strasbourg, Conseil de l'Europe.

COLIN G. 2012. Construction du bi-plurilinguisme en français langue de scolarisation: apprentissage d'une L2 en enseignement bilingue précoce. Thèse partiellement en ligne. Université Montpellier 3. http://www.theses.fr/2012MON30047/document. CLERC J.-F. 2016. L'impossible équation, Dermières Nouvelles d'Alsace, 28.5, 13.

DUVERGER J. 2009. Conférence en ligne - Sćminaire bilingue du GIEP. DMotionInfo.

http://www.dmotioninfo.com/video/11666691/conference-dejean-duverger-seminaire-bilin

GAJO L., BERTHOUD A.-G. 2008. Construction intégrée des savoirs linguistiques et disciplinaires dans l'enseignement bilingue au secondaire et au tertiaire. Rapport final PNR 56. Berne: Fonds national suisse de la recherche scientifique. Téléchargeable sur le site www.pnr56.ch 


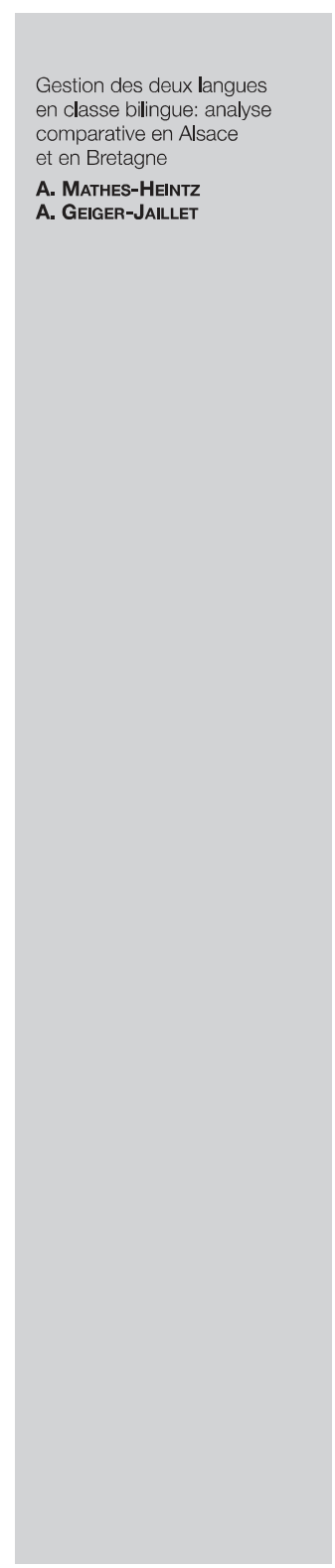

GEIGER-JAILLET A. 2016. Les facteurs d'échec et de réussite dans le bilinguisme précoce: les apports de Jules Ronjat revisités à la lumière des 100 ans de sa publication, 85-102, in Escudié P. (coord.): Autour des travaux de Jules Ronjat, 1913-2013. Unité et diversité des Langues. Théorie et pratique de l'acquisition bilingue et de l'intercompréhension. ESPE de l'Académie de Toulouse/Université Toulouse 2/ DGLFLF.

GEIGER-JAILLET A. 2005. Le bilinguisme pour grandir: naître bilingue ou le devenir par l'école. Paris: L'Harmattan.

HÉLOT C., RUBIO M.-N. 2013. Développement du langage et plurilinguisme chez le jeune enfant. Toulouse: Erès.

KAIL M. 2015. L'acquisition de plusieurs langues. Paris: PUF.

LÜDI G. 2004. Pour une linguistique de la compétence du locutcur plurilingue. Revue française de linguistique appliquée 2/2004 (Vol. IX), 125-135, http://www.cairn.info/revue-francaise-de-linguistiqueappliquee-2004-2-page-125.htm.

MATHES-HEINTZ A. 2017. Pratiques enseignantes concernant la gestion des deux langues dans les situations d'apprentissage en L2. Analyse comparative des professeurs des écoles bilingues en Alsace et en Bretagne. Université de Strasbourg. (Mémoire de master PIF TFE non publié).

Ministère de l'Education Nationale (MEN) 2001. Modalités de mise en ceuvre de l'enseignement bilingue à parité horaire. B.O. N³3 (13 septembre). PETIT J. 2001. L'immersion, une révolution. Colmar: Jérôme Do Bentzinger.

STEFFEN G. 2013. L.es disciplines dans l'enseignement bilingue. Frankfurt am Main: Peter Lang. 